\title{
Alternative Discriminating Function for Determining Hormone Dependency of Breast Cancer
}

\author{
H. MILLER,* M.D., M.R.C.P., M.C.PATH. ; J. A. DURANT,* B.SC. ; A. G. JACOBS,* M.B., B.S. \\ JACQUELINE F. ALLISON,* A.I.M.L.T.
}

Brit. med. F., 1967, 1, 147-149

It is common practice to consider performing hypophysectomy or adrenalectomy combined with oophorectomy on women suffering from metastatic breast cancer. Satisfactory, albeit temporary, improvement can be expected in only about onehalf of those operated on, so that it would be advantageous if it were possible to predict whether a useful response could be anticipated. Accurate prediction would prevent patients being subjected to a major operation from which no benefit could be expected.

By studying the urinary steroid metabolites of a number of these patients before one or other of these operations were performed, and relating the results obtained to the clinical response after operation, Bulbrook, Greenwood, and Hayward (1960) were able to derive a function which discriminated between the responsive and non-responsive groups. This discriminant function was calculated as $80-80$ (17-OHCS (mg./24 hours)) + aetiocholanolone ( $\mu$ g. $/ 24$ hours). 17-OHCS (17-hydroxycorticosteroids or total 17-oxogenic steroids) were measured by the method of Appleby, Gibson, Norymberski, and Stubbs (1955), and aetiocholanolone ( $3 \alpha$-hydroxy-5 $\beta$-androstan-17-one) by the method of Kellie and Wade (1957). A positive numerical result was obtained in the responsive patients and a negative result in the non-responsive group.

When this function was determined in a series of normal women and women suffering from non-metastatic breast cancer it was shown that, whereas it was unusual to find a negative discriminant in normal women under 65 , over half of the early breast cancer cases gave negative discriminants (Bulbrook, Hayward, Spicer, and Thomas, 1962b).

The British Medical fournal (1965) recognized the possible clinical significance of this discriminant function even though its physiological meaning was unknown.

There has been little published by other laboratories in evaluation of this work, and this apparent lack of interest may be due to the technical difficulties of estimating urinary aetiocholanolone and to the amount of working time needed to do so.

The three major components of the 11-deoxy-17-oxosteroid group of urinary steroids are aetiocholanolone, androsterone ( $3 \alpha$-hydroxy- $5 \alpha$-androstan-17-one), and dehydroepiandrosterone (3 $\beta$-hydroxy-androst-5-en-17-one). Bulbrook et al. (1960) found that androsterone was almost as useful as aetiocholanolone for that purpose, whereas dehydroepiandrosterone was not.

It is comparatively simple to measure this group of steroids in urine, and they are derived mainly from the metabolism of adrenocortical androgenic hormones. It was decided to attempt discrimination, therefore, by estimating the 17-OHCS and the unfractionated 11-deoxy-17-oxosteroids. Both these analyses are within the capabilities of laboratories accustomed to estimating urinary steroid groups for the more usual clinical indications.

In order to compare any alternative discriminant with the original it was necessary to estimate aetiocholanolone. A new

* Portsmouth and I.O.W. Area Pathological Service, Department of Pathology, Royal Portsmouth Hospital, Portsmouth. method was devised for this purpose which did not need the complicated and expensive apparatus of previously published methods (Miller and Durant, 1966).

The results recorded in this paper show a satisfactory correlation between the original discriminant and an alternative function obtained from the expression 11-deoxy-17-oxosteroids (mg. per 24 hours)/17-OHCS (mg. per 24 hours).

This alternative discriminant is being used in this laboratory in parallel with the original discriminant to study cases of breast cancer in the early and late stages of the disease. The accuracy of discrimination will be reported in due course, but it was thought that prior publication of the means of determining the alternative discriminant would be of interest to other clinical laboratories.

\section{Methods}

A single 24-hour collection of urine was obtained from each of a group of normal women and from each of the patients suffering from breast cancer and referred to us by the surgeons and radiotherapists of the Portsmouth Group of Hospitals. The cancer cases were accepted whatever the stage of the disease, but no urine collections were examined from those who had undergone mastectomy less than six weeks previously or were having hormones by mouth. Most of the stage 1 and 2 cancer subjects collected their urine before mastectomy. Those with metastatic cancer were usually referred to us before hormones were administered. A small number of the latter already undergoing hormone treatment ceased this treatment for one week before making their urine collection. The urine samples were stored at $4^{\circ}$ C. without preservative until they could be analysed.

Total 17-OHCS were estimated by the method of Metcalf (1963), which employs periodate oxidation of the corticosteroids and expresses the results in terms of an internal cortisol standard. In our hands this method gave almost identical results to that of Appleby et al. (1955), which uses bismuthate oxidation, the results being calculated in terms of a dehydroepiandrosterone external standard.

11-deoxy-17-oxosteroids were measured by the simple column chromatographic method of Goldzieher and Axelrod (1962), and aetiocholanolone was measured by thin-layer chromatography as described by Miller and Durant (1966). Treatment of the urine before the last two determinations and the particular modification of the Zimmerman reaction used for measuring the 17-oxosteroids are also described in the latter paper.

\section{Results}

This paper describes the results obtained from 94 women with breast cancer and 55 healthy women. Only the alternative discriminant was obtained for the first 23 cases of cancer, since it was not at first possible to estimate aetiocholanolone with the 
available facilities. In the remaining women both the alternative and original discriminants were calculated.

Frequency distribution of the alternative discriminant in both groups is shown in Fig. 1. The relation of this function with age is plotted in Figs. 2 and 3.

Values obtained for both discriminants are compared in Figs. 4 and 5. In both the healthy and the cancer groups there are a small number of women whose aetiocholanolone excretion is less than $30 \%$ of their total 11-deoxy-17-oxosteroids. This apparently arbitrary subdivision was made because it became clear that these women fitted a calculated regression line different from that of the main body of the group.
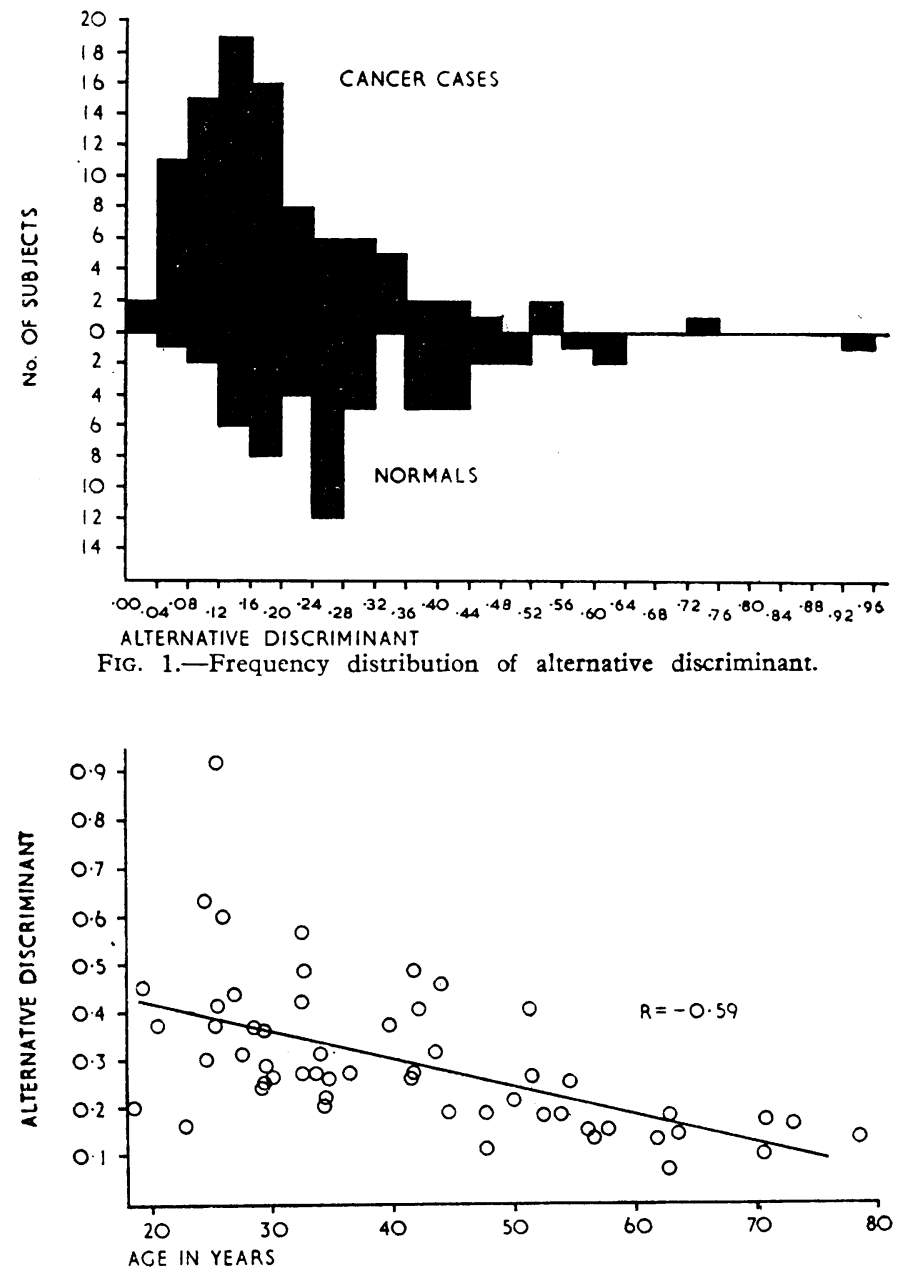

FIG. 2.-Correlation of alternative discriminant with age in healthy women.

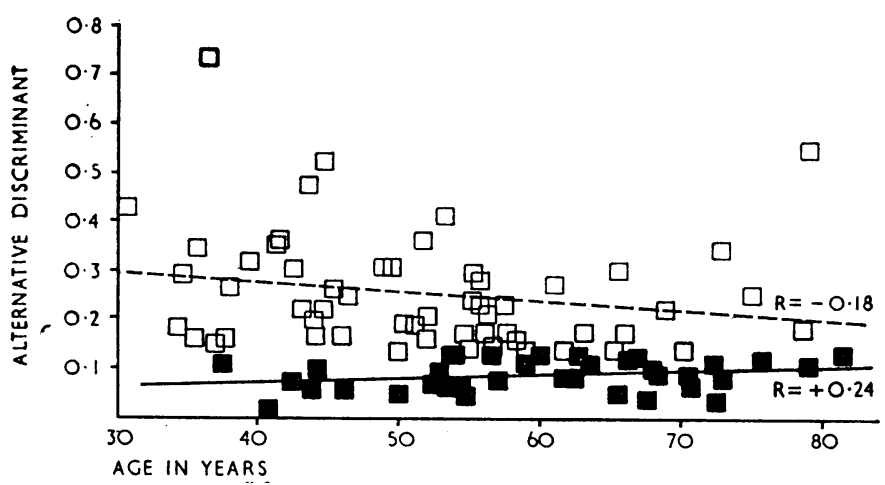

FIG. 3.-Correlation of alternative discriminant with age in the cancer cases. $\square=$ Discriminant greater than 0.13 (positive). $\quad=$ Discriminant less than 0.13 (negative).

\section{Discussion}

The factors determining whether or not an individual case of breast cancer is hormone-dependent are not known. It is clear that the breast is a target organ for a variety of hormones of pituitary, ovarian, and perhaps adrenocortical origin. The difference between the hormone-responsive and non-responsive tumours may be due to a difference in the hormonal milieu interieur, or it may be that no such difference occurs but the non-responsive tumours have escaped the control of normal

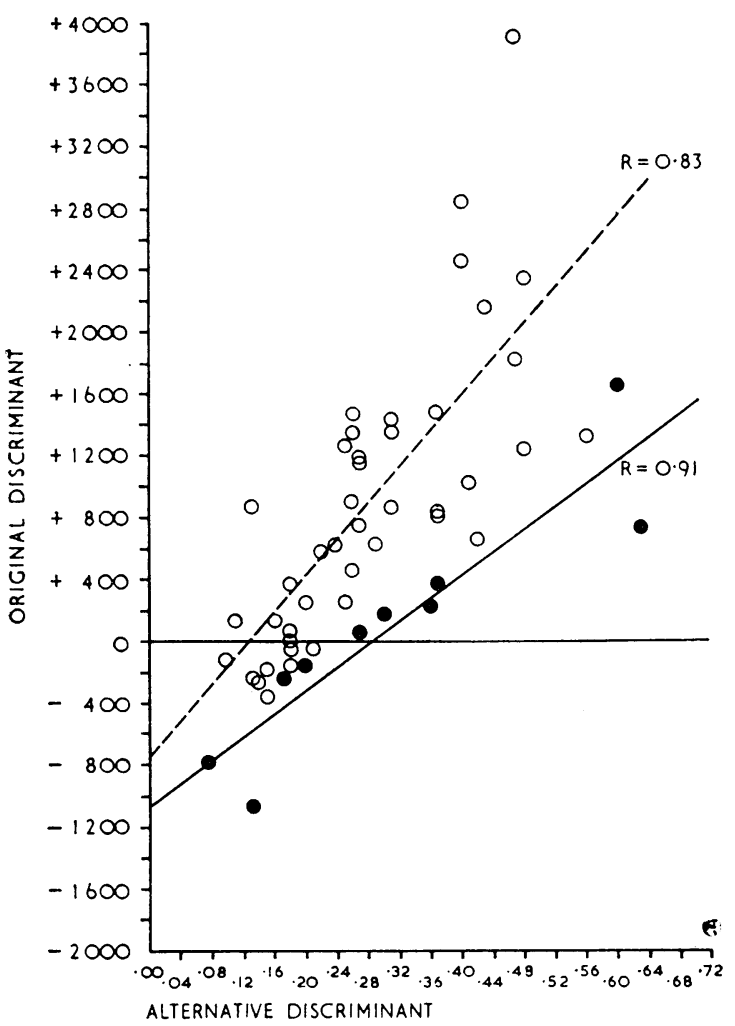

FIG. 4.-Correlation between the alternative and original discriminant in the healthy women. $\mathrm{O}=$ Women with aetiocholanolone excretion greater than $30 \%$ of total 11deoxy-17-oxosteroids. $=$ Women with aetiocholanolone excretion less than $30 \%$ of total 11-deoxy-17-oxosteroids.

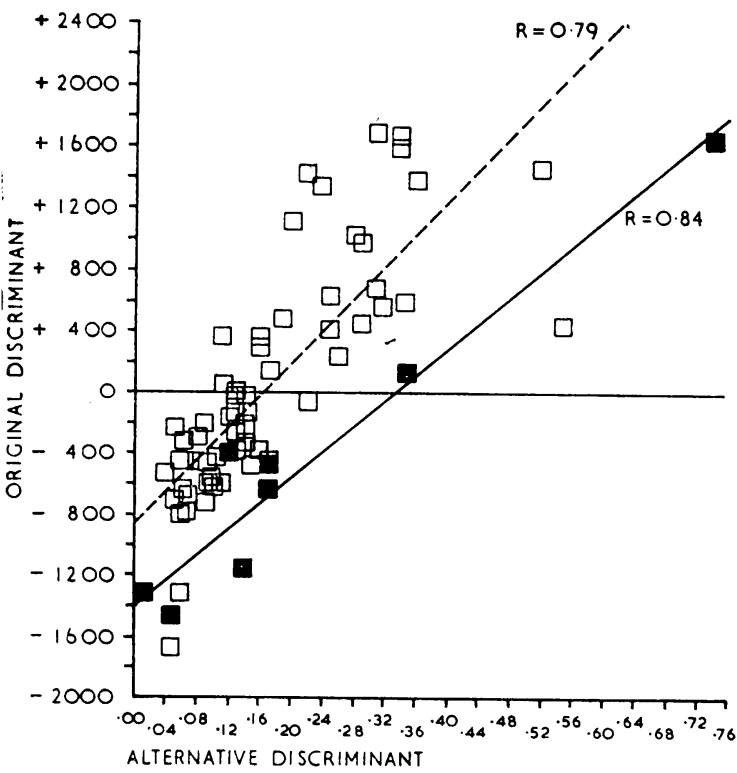

Fig. 5.-Correlation between the alternative and original discriminant in the cancer cases. $\square=$ Women with aetiocholanolone excretion greater than $30 \%$ of total 11-deoxy17-oxosteroids. $=$ Women with aetiocholanolone excretion less than $30 \%$ of total 11-deoxy-17-oxosteroids. 
mammary hormonal influences. The inevitable deterioration after initially successful hormonal treatment shows that the latter situation arises sooner or later even if the former postulate is true.

The work of Bulbrook et al. (1960) and the studies of Atkins, Falconer, Hayward, MacLean, Schurr, and Armitage (1960) indicate that a satisfactory response to adrenalectomy or hypophysectomy can be expected when androgenic urinary metabolites are high in comparison with the urinary corticosteroid metabolites. Together with the studies of Bulbrook et al. (1962a, and 1962b), there is considerable evidence to suggest that at least some cases of breast cancer in both the early and the late stages occur in a hormonal situation which deviates from the normal when judged by the exereted urinary hormonal metabolites, and these cases are less responsive to endocrine organ oblation.

The results obtained in this study (Fig. 1) with use of the alternative discriminant confirms that an unselected series of cases of breast cancer (subject to the conditions mentioned in the methods section) differ from a group of normal women in the excretion of androgenic metabolites compared with corticosteroid metabolite excretion. This difference is somewhat biased, since it can be seen from Figs. 2 and 3 that the value of the alternative discriminant is related in some degree to age, and the normal group contained many more young women than the cancer group. It seems clear, however, from inspection of Figs. 2 and 3 that, whereas only two of the normal women aged under 60 had an alternative discriminant less than $0.14,20$ of the women with breast cancer and under 60 years of age were found to have alternative discriminants below this figure. This conclusion is emphasized by the calculated regression lines shown in these figures. While the normal women and the cancer cases. with alternative discriminants above 0.14 show a negative correlation with increasing age, the cancer cases with alternative discriminants less than 0.14 have a slightly positive correlation. These discriminant: age correlations are similar to those of Bulbrook et al. (1962a, 1962b), who related the original discriminant with age for normal women and for women with early and advanced breast cancer. In all these series of breast cancer, therefore, the lowest discriminants are found in the youngest patients. It is tempting to suggest that this reflects the poorer overall prognosis of the younger afflicted women.

It is also apparent (Figs. 4 and 5) that the alternative discriminant correlates closely with the original discriminant function. These results show that an alternative discriminant of 0.13 in most of the normal group and 0.16 in most of the cancer group is equivalent to the zero value of the original discriminant-that is, a positive alternative discriminant is greater than 0.16 and a negative alternative discriminant is less than 0.13. A small group of women in both groups, however, show a negative original discriminant when they have a positive alternative discriminant. The prognostic significance of this discrepancy remains to be explained, but in all of these women the aetiocholanolone excretion is less than $30 \%$ of the total $11-$ deoxy-17-oxosteroid excretion. There is evidence (B. S.
Thomas, personal communication, 1965) that aetiocholanolone excretion is decreased relative to the 11-deoxy-17-oxosteroid excretion during and for some while after states of hyperthyroidism whether endogenously or therapeutically induced. In one of our normal subjects the two discriminants were estimated repeatedly throughout one menstrual cycle, and while the alternative discriminant remained positive the original discriminant fluctuated repeatedly between positive and negative results. In this subject the aetiocholanolone excretion was always less than $30 \%$ of the total 11-deoxy-17-oxosteroid excretion. The results in the same subject first suggested the need to subdivide each group of women by this relationship of aetiocholanolone to total 11-deoxy-17-oxosteroids.

It is clear that in most women there is excellent correlation between the two discriminants, so that the more easily obtained alternative method should prove of equal value to the original method. It may prove to be of more value in the small group of women discussed above.

\section{Summary}

A successful attempt to discriminate between responsive and non-responsive cases of advanced breast cancer to endocrine organ ablation has been reported. This necessitates the estimation of urinary aetiocholanolone, a difficult task for clinical chemical laboratories.

This paper describes an alternative function which does not require fractionation of the 11-deoxy-17-oxosteroids and can be determined by any clinical laboratory accustomed to assaying urinary steroids for the more usual clinical indications.

Results are presented which show good agreement between the new and the original discriminant except in a small group of women who excrete subnormal amounts of aetiocholanolone in relation to the total androgenic urinary output.

Our thanks are due to Professor W. Klyne, of the M.R.C. Steroid Reference Collection, for allowing us to have small quantities of pure steroids. We have also to thank the members of the Royal Portsmouth Hospital staff who so kindly provided us with 24-hour urine samples. We are very grateful to Tenovus for generous financial assistance, which is allowing us to proceed with this work.

\section{REFERENCES}

Appleby, J. I., Gibson, G., Norymberski, J. K., and Stubbs, R. D. (1965). Biochem. 7., 60, 453.

Atkins, H. J. B., Falconer, M. A., Hayward, J. L., MacLean, K. S., Schurr, P. H., and Armitage, P. (1960). Lancet, 1, 1148.

Brit. med. F., 1965, 2, 1198. Bulbrook, R., D., Greenwood, F. C., and Hayward, J. L. (1960). Lancet,
1, 1154.

Hayward, J. L., Spicer, C. C., and Thomas, B. S. (1962a). Ibid., 2, 1235 .

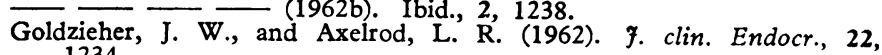
1234.

Kellie, A. E., and Wade, A. P. (1957). Biochem. F., 66, 196.

Metcalf, M. G. (1963). F. Endocr., 26, 415.
Miller, H., and Durant, J. A. (1966). Clin. chim. Acta, 14, 475. 